\title{
Reassortant highly pathogenic avian influenza H5N6 virus originating from the wildbird-origin H5N6 and the poultry H9N2/H7N9 viruses in Xinjiang, China*
}

\author{
QIAN ZHANG ${ }^{1}, X^{\prime N D I}$ MEI $^{1}$, CHENG ZHANG ${ }^{1,2}$, FEI DU ${ }^{1}$, NANA CHANG ${ }^{1}$, \\ DILIHUM AJI ${ }^{1}$, JUAN LI ${ }^{3}$, WEIFENG SHI ${ }^{3}$, YUHAI BI ${ }^{1,2}$, ZHENGHAI MA ${ }^{1}$
}

\begin{abstract}
${ }^{1}$ Xinjiang Key Laboratory of Biological Resources and Genetic Engineering, College of Life Science and Technology, Xinjiang University, Urumqi, 830046, China

${ }^{2}$ CAS Key Laboratory of Pathogenic Microbiology and Immunology, Collaborative Innovation Center for Diagnosis and Treatment of Infections Disease, Institute of Microbiology, Center for Influenza Research and Early-Warning (CASCIRE), University of the Chinese Academy of Science, Beijing, 100101, China

${ }^{3}$ Key Laboratory of Etiology and Emerging Infections Disease in Shandong First Medical University, Taian, 271016, China
\end{abstract}

Zhang Q., Mei X., Zhang C., Du F., Chang N., Aji D., Li J., Shi W., Bi Y., Ma Z.

Reassortant highly pathogenic avian influenza H5N6 virus originating from the wildbird-origin H5N6 and the poultry H9N2/H7N9 viruses in Xinjiang, China

\section{Summary}

The asian lineage of $\mathrm{H5}, \mathrm{H7}$, and $\mathrm{H9}$ avian influenza viruses (AIVs) is the most prevalent in the world, and the co-existence of $\mathrm{H5}, \mathrm{H7}$, and $\mathrm{H9}$ viruses provide an opportunity to generate some novel reassortants. Herein we report our isolation of two H5N6 virus strains (named XJ-H5N6) from domestic geese in live poultry markets (LPMs) in Urumqi during the winter of 2016-2017, and analysis of the viral genetic mutations, pathogenicity, reassortant, and origin. BLAST analysis revealed that the $H A, N A, M P, N P$, and $N S$ of XJ-H5N6 had the highest sequence identity $(98.5-99.5 \%)$ with those 5 genes from H5N6 highly pathogenic avian influenza virus (HPAIV) isolated from wild birds in Ningxia (NX-WB-H5N6); the viral $P A$ and $P B 1$ had the highest sequence identity (98.6\% and $98.9 \%)$ with those 2 genes from HPAIV strain A/Environment/Chongqing/45373/2015 (H5N6) (CQ-EN-H5N6); while the viral $P B 2$ had the highest sequence identity $(99.0 \%)$ with this gene from low pathogenic avian influenza virus (LPAIV) strain A/environment/Jiangsu/01.20 TCCX004/2015 (H9N2). Except for the $P B 2$, phylogenetic analysis showed that the other 7 viral genes were clustered together with NX-WB-H5N6 and CQ-EN-H5N6, while the viral $P B 2$ was clustered together with the poultry H9N2/H7N9 viruses. The viral HA protein contained multiple basic amino acid residues (RRRKR/GL) at the cleavage site which represents HPAIV. Additionally, the viral multiple mutations could enhance the viral virulence and the capacity of cross-species transmission. Our data indicate that XJ-H5N6 has been generated by the reassortant of H5N6 HPAIV from wild birds in Ningxia and H9N2/H7N9 LPAIV in poultry. The results of this study may help us understand the viral adaptation and transmission patterns from wild birds to poultry.

Keywords: avian influenza virus, H5N6, poultry, wild birds, reassortant

Since the emergence of H5N1 (A/goose/Guangdong/ 1/1996) in China in 1996, the viral $H A$ has evolved into multiple distinct phylogenetic clades, subclades, and lineages around the globe. In 2014, the clade 2.3.4.4 H5Nx HPAIVs spread rapidly via migratory birds and evolved into four groups (A-D). A group C H5N6 outbreak was initially reported in Laos in 2013, then spread rapidly to Vietnam, Japan, South Korea, and China (26). In China, H5N6 viruses have widely

* This work was supported by Xinjiang Uygur Autonomous Region Regional Cooperative Innovation Project (2017E01022) and the National Science Technology Major Project (2018ZX10101004). circulated in wild waterfowl and poultry (1). A previous study showed that the H5N6 AIVs in poultry can transmit among ferrets via direct contact (23). H5N6 HPAIVs with the same genotype to human-origin strains have also been found in apparently healthy wild birds (2). So the virus can be transmitted through migratory birds, and almost all H5N6 patients have documented exposure to poultry and/or LPMs $(1,8)$. Therefore, surveillance for AIVs is needed in commercial poultry.

Urumqi is located in the northern Tianshan Mountain, which is an important habitat for migratory 
birds (13). In this area, a lot of reservoirs have been formed by the construction of a dam in the narrow mouth of a ravine or a river originating from melting snow (27). These reservoirs are an important habitat for wild birds, and are also used as waterfowl farms. There is a high probability of contact between wild birds and waterfowls. In fact, HPAIVs outbreaks in Xinjiang have been repeatedly documented in recent years, and there have been several outbreaks of H5N6 HPAIV in Xinjiang between 2019 and 2020. In the current study, we isolated the H5N6 HPAIVs in poultry from LPMs in Urumqi, and analyzed the origin and molecular characteristics of the virus.

\section{Material and methods}

Samples collection and storage. From October 2016 to April 2017, a total of 354 oropharyngeal and cloacal swabs from apparently healthy poultry (chickens, ducks, and geese) in LPMs in Urumqi, China were collected and placed into tubes containing viral transport medium DMEM $(1,000 \mu / \mathrm{L}$ penicillin, $500 \mu \mathrm{g} / \mathrm{L}$ streptomycin, $100 \mathrm{mg} / \mathrm{L}$ Nystatin, $100 \mathrm{mg} / \mathrm{L}$ Polymyxin B sulfate salt, $1000 \mathrm{mg} / \mathrm{L}$ Sulfamethoxazole, $0.05 \mathrm{~g} / \mathrm{L}$ Ofloxacin). These tubes were kept in an icebox before transport to the laboratory, and then immediately stored at $-80^{\circ} \mathrm{C}$.

Virus isolation and Whole-Genome Sequencing. The solid debris in the swabs was pelleted by centrifugation at $7000 \times \mathrm{g}$ for $5 \mathrm{~min}$. The supernatants were inoculated into the allantoic cavities of 10-day-old specific-pathogen-free (SPF) embryonated chicken eggs (Beijing Merial Ltd). After incubation at $37^{\circ} \mathrm{C}$ for $48-72 \mathrm{~h}$, the allantois fluids of the inoculated eggs were collected and tested by Hemagglutination assay, the positive samples were further confirmed by RT-PCR using the universal influenza A primers target the PB1 gene (24).

The whole-genome sequences of AIV isolates were determined by Next-generation sequencing (NGS), the viral RNAs were quantified using the 2100 Bioanalyzer System (Agilent Technologies). RT-PCR and cDNA synthesis were conducted using the PrimeScript One Step RT-PCR kit (Takara) with influenza A-specific primers MBTuni-12 and MBTuni-13 (1, 29). The sequencing libraries with an insert size of $200 \mathrm{bp}$ were prepared by end-repairing, dA-tailing, adaptor ligation, and PCR amplification, all according to the instructions provided by the manufacturer (Illumina). The libraries were sequenced on an Illumina HiSeq 4000 Sequencer by $101 \mathrm{bp}$ paired-end sequencing, and sequencing depth for AIV isolates was $0.2 \mathrm{G}$ per sample.

Phylogenetic analysis and molecular characterization. The sequences of AIVs were obtained from the Global Initiative on Sharing All Influenza Data (GISAID) and NCBI. The sequences were then aligned using the Clustal $\mathrm{W}$ program implemented in MEGA 7.0, and then Phylogenetic trees were constructed using MrBayes v3.2.6. The generalized time-reversible model parameters and priors were incorporated into the nexus file for execution in MrBayes, utiliz- ing subtree pruning and redrafting branch swapping and the GTR+ Gamma model of nucleotide substitution for each of the viral genes. The programs PAUP version 4.0, jModelTest were used. Monte Carlo Markov chains were run for 1 million iterations, with a sampling frequency of 10,000 steps, the tree was constructed after discharging the first $10 \%$ burn-in. Finally, the convergence standard deviation was assessed by estimating split frequent $(<0.01)$, the tree was visualized using FigTree v1.4.2. MegAlign in Lasergene 7 (DNASTAR) was used to determine the nucleotide sequence similarity percentages and mutation sites.

\section{Results and discussion}

Virus detection, isolation, and subtyping. Through the regular AIV surveillance in Urumqi LPMs from October 2016 to April 2017, 9 strains of AIV were isolated (7 as H5N8 viruses and 2 as H5N6 viruses) from 354 samples (Supplementary Tab. 1). 2 strains of $\mathrm{H} 5 \mathrm{~N} 6$ virus were isolated from two domestic geese on December 18, 2016, one from a cloaca swab and another from a throat swab. The 2 viruses were the focus of this paper. The sequence alignment analysis showed the viral 8 genes (including $H A, N A, P B 1$, $P B 2, N S, P A, N P$, and $M P$ ) of the 2 isolates shared very high nucleotide identity $(>99.9 \%)$ respectively, the isolates were named A/Goose/Xinjiang/008/2016 (H5N6) and A/Goose/Xinjiang/009/2016 (H5N6), or XJ-H5N6 for short. The full sequences of XJ-H5N6 viruses were submitted to GISAID (accession nos.: EPI1548859-1548874).

Nucleotide identity analysis. BLAST analysis was performed for each gene fragment of the isolates (Tab. 1). The $H A$ and $N P$ had the highest sequence identity with those 2 genes from A/Shoveller/Ningxia/ 483-28/2015 (H5N6) respectively. The $N A$ has the highest sequence identity with this gene from A/Common Teal/Ningxia/488-53/2015 (H5N6) (NX488-53). The $M P$ and NS had the highest sequence identity with those 2 genes from A/White-eyed Pochard/ Ningxia/486-33/2015 (H5N6), respectively, hence all the 5 genes had the highest sequence identity with those genes from NX-WB-H5N6. The $P A$ and $P B 1$ had the highest sequence identity with those 2 genes from $\mathrm{A} /$ Environment/Chongqing/45373/2015 (H5N6) (CQ-EN-H5N6), respectively. Additionally, the $P B 2$ has the highest sequence identity with
Tab. 1. The highest nucleotide identity with the gene of XJ-H5N6 virus

\begin{tabular}{|l|l|l|c|}
\hline \multicolumn{1}{|c|}{ Gene } & \multicolumn{1}{|c|}{ Viruses with the highest nucleotide identity } & Accession no. & Identity (\%) \\
\hline HA & A/Shoveller/Ningxia/483-28/2015 (H5N6) & EPI1159893 & 98.5 \\
NA & A/Common Teal/Ningxia/488-53/2015 (H5N6) & EPI1159965 & 98.8 \\
MP & A/Ruddy Shelduck/Ningxia/486-33/2015 (H5N6) & EPI1159753 & 99.0 \\
NP & A/Shoveller/Ningxia/483-28/2015 (H5N6) & EPI1159954 & 99.1 \\
NS & A/Ruddy Shelduck/Ningxia/486-33/2015 (H5N6) & EPI1159954 & 99.5 \\
PA & A/Environment/Chongqing/45373/2015 (H5N6) & EPI749549 & 98.9 \\
PB1 & A/Environment/Chongqing/45373/2015 (H5N6) & EPI749551 & 98.6 \\
PB2 & A/environment/Jiangsu/01.20 TCCX004/2015(H9N2) & EPI660256 & 99.0 \\
\hline
\end{tabular}



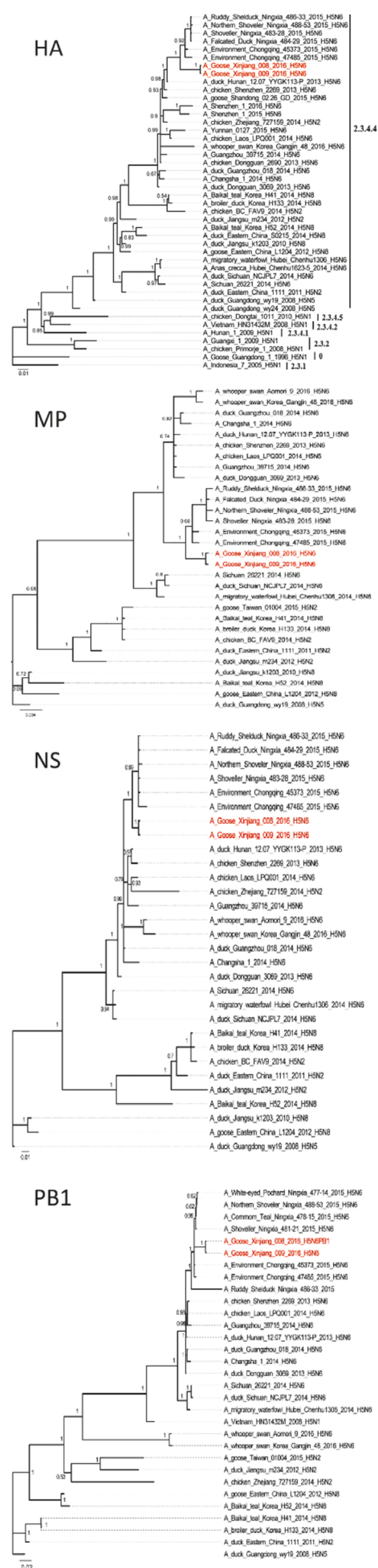

Fig. 1. Phylogenetic analysis of $H A, N A, M P, N P, N S, P A, P B 1, P B 2$ of XJ-H5N6. The H5N6 viruses isolated in this study are colored in red

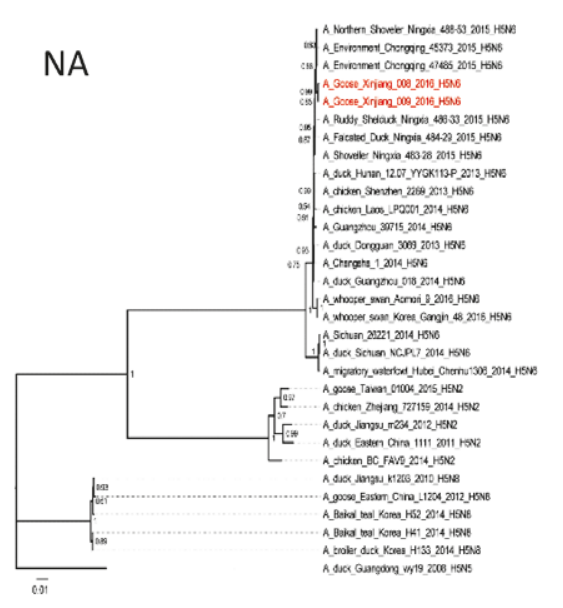

this gene from A/environment/ Jiangsu/01.20TCCX004/2015 (H9N2).

Phylogenetic analysis. Phylogenetic analyses were performed on each gene of XJ-H5N6 (Fig. 1). $H A$ of XJ-H5N6 was clustered together with NX-WB-H5N6 and CQ-EN-H5N6, and belonged to Group $\mathrm{C}$ of clade 2.3.4.4. The $N A, M P, N P, N S, P A$, and $P B 1$ were also clustered together with NX-WB-H5N6 and CQ-EN-H5N6 viruses. Nevertheless, the $P B 2$ was clustered together with A/environment/Jiangsu/TCCX004/2015 (H9N2) and A/chicken/Jiangsu/ SD012/2015 (H7N9), and not together with $\mathrm{H} 7 \mathrm{~N} 9$ isolated from Xinjiang including A/chicken/ Xinjiang/SD033/2014 (H7N9), A/chicken/Xinjiang/SD016/2014 (H7N9), and A/Environment/ Xinjiang/73033/2013 (H7N9). In brief, the viral $P B 2$ originated from the poultry H9N2/ H7N9 viruses in Jiangsu, and the other 7 viral segments originated from NX-WB-H5N6 or CQ-ENH5N6. Moreover, all the viral genes also had high sequence identity (98.0-99.2\%) and a small genetic distance with $\mathrm{A} / \mathrm{duck} /$ Hunan/12.07 YYGK113-P/2013 (H5N6) (HN113-P). HN113-P was thought to be the origin of NX-WB-H5N6, except for the $P B 1$, while the 7 genes of NX-WBH5N6 originated from HN113-P and share the highest nucleotide identity with EN-CQ-45373 (12). Therefore, XJ-H5N6 is a reassortant virus that may originate from NX-WB-H5N6 and the poultry $\mathrm{H} 9 \mathrm{~N} 2 / \mathrm{H} 7 \mathrm{~N} 9$ viruses in Jiangsu.

The avian-origin $\mathrm{H} 9 \mathrm{~N} 2$ viruses widely exist in wild waterfowls and poultry and under certain circumstances can be transmitted to humans, usually causing mild infections. It is also known that several new emerging zoonotic AIVs such as H7N9, H5N1, $\mathrm{H} 10 \mathrm{~N} 8$, and H5N6 contain the $P B 2$ from the H9N2 viruses (5), and this virus can frequently donate the internal genes to generate 
reassortant viruses for adaption in new hosts (25). For example, the H5N1 HPAIVs from a human in America is a reassortant with the $P B 2$ from an $\mathrm{H} 9 \mathrm{~N} 2$ virus (18), and the internal genes of the novel human-pathogenic H7N9 AIVs are also derived from $\mathrm{H} 9 \mathrm{~N} 2$ viruses circulating in Eastern China (7). Influenza virus adaptive mutations in $P B 2$ usually cause enhanced polymerase activity and promote high virulence (22). And our data indicate that H9N2/H7N9 LPAIV in poultry donate $P B 2$ to generate the reassortant viruses XJ-H5N6, which could adapt in poultry.

Molecular characteristics. $H A, N A, P B 2$, and $N S 1$ are known as major determinants of the host range restriction and pathogenicTab. 2. The mutation of XJ-H5N6 virus

\begin{tabular}{|l|l|l|c|}
\hline Protein & \multicolumn{1}{|c|}{ Mutation } & \multicolumn{1}{|c|}{ Function } & Reference \\
\hline HA & S128P & Enhancing binding to human-like $\alpha 2,6-S A$ receptor & $(22)$ \\
& S137A & & $(22)$ \\
& T160A & & $(22)$ \\
& Q226L & Avian-like $\alpha 2,3-S A$ receptor & \\
& G228S & & \\
& $\begin{array}{l}\text { Cleavage site } \\
\text { RERRRKR/G }\end{array}$ & Highly pathogenic & \\
\hline NA & 59-69 del. & Increasing virulence in mice; adapted to poultry & $(3),(4),(15),(17)$ \\
\hline PB2 & L89V & Enhancing viral virulence and replication in mice & $(10)$ \\
& G309D & & $(10)$ \\
& R477G & & $(10)$ \\
& I495V & & $(10)$ \\
& I504V & & $(20)$ \\
& D391E & Increasing viral replication and transmission in mammals & $(16)$ \\
& A661E & & $(16)$ \\
\hline \multirow{2}{*}{ NS1 } & D92E & Highly pathogenic and increasing the virulence in mice & $(21)$ \\
& P42S & Increasing viral replication and virulence in mice & $(6)$ \\
\hline
\end{tabular}
ity of influenza viruses $(11,17)$.

In our study, the multiple mutations of the isolates could help us understand the viral risk, as well as adaptation and transmission patterns from wild bird to poultry (Tab. 2). XJ-H5N6 carried the poly-basic amino acids (RRRKR/GL) at the cleavage site in HA, which represents HPAIV. The receptor-binding site contains Q226 and G228 (H3 numbering), suggesting an avian-like $\alpha 2,3$-sialic acid $(\alpha 2,3-\mathrm{SA})$ receptorbinding preference, and the S128P, S137A and T160A mutations in the HA could enhance binding capacity to a human-like $\alpha-2,6-\mathrm{SA}$ receptor (21). These 3 mutations were also found in A/Yunnan/0127/2015 (H5N6) from human infection and NX488-53, and it has been reported that $\mathrm{NX} 488-53$ virus can infect $\mathrm{BALB} / \mathrm{c}$ mice and transmit among guinea pigs via direct contact or through aerosol $(12,28)$.

Here we found that the NA stalk of XJ-H5N6 possessed an 11-amino-acid deletion (59-69 deletion) (Tab. 2), which might enable wild bird-origin AIVs to adapt to domestic poultry and influence the viral virulence. Given previous reports that the short-stalk NA of H7N9 (3) and H5N1 (14) AIVs could increase virulence in mice, and also are characteristic of the adaptation of influenza viruses from poultry (16). Additionally, the adaptation of AIV with short-stalk NA can cross from wild aquatic birds to poultry (4).

In the current study, several mutations in $P B 2$ (Tab. 2), including L89V, G309D, R477G, I495V, I504V, D391E, and A661E, could play essential roles in enhancing the viral virulence. Our findings are also in agreement with previous findings in that the L89V, G309D, R477G, and I495V mutations increased the viral replication activity in mammalian cells and increase viral virulence in mice (9), the I504V mutation enhanced viral polymerase activity and virulence

in mice (19), and the D391E and A661E mutations induced favorable conditions for the viral replication and transmission in mammalian hosts by affecting the PB2 structure (15).

With respect to NS1 that plays an important role in virus replication, virulence, and inhibition of the host antiviral immune response, it is known that certain NS1 mutations are responsible for alterations in virus pathogenicity (10). Indeed, 2 among those NS1 mutations, D92E and P42S, have drawn a great deal of attention. In particular, the D92E mutation is in association with the resistance to the antiviral effects of cytokines and a high fatality rate (20), and the P42S mutation plays an important role in regulating the host IFN response by blocking the activation of IRF3 and thus facilitates virus replication (6). Therefore, the $\mathrm{D} 92 \mathrm{E}$ and $\mathrm{P} 42 \mathrm{~S}$ mutations in the NS1 (Tab. 2) could enhance the viral virulence.

In summary, our data indicate that XJ-H5N6 is a novel reassortant HPAIV and has originated from H5N6 HPAIV in wild birds and H9N2/H7N9 LPAIV in poultry. The multiple mutations in the virus could help us to understand the viral adaptation and transmission patterns from wild bird to poultry, and assess the risk of an influenza pandemic and cross-species infection.

\section{Reference}

1. Bi Y., Chen Q., Wang Q., Chen J., Jin T., Wong G., Quan C., Liu J., Wu J., Yin R., Zhao L., Li M., Ding Z., Zou R., Xu W., Li H., Wang H., Tian K., Fu G., Huang Y., Shestopalov A., Li S., Xu B., Yu H., Luo T., Lu L., Xu X., Luo Y., Liu Y., Shi W., Liu D., Gao G. F.: Genesis, evolution and prevalence of H5N6 avian influenza viruses in China. Cell Host Microbe 2016, 20, 810-821.

2. Bi Y., Liu H., Xiong C., Di Liu., Shi W., Li M., Liu S., Chen J., Chen G., Li Y., Yang G., Lei Y., Xiong Y., Lei F., Wang H., Chen Q., Chen J., Gao G. F.: Novel avian influenza A (H5N6) viruses isolated in migratory waterfowl before the first human case reported in China, 2014. Sci. Rep. 2016, 6, 29888.

3. Bi Y., Xiao H., Chen Q., Wu Y., Fu L., Quan C., Wong G., Liu J., Haywood J., Liu Y., Zhou B., Yan J., Liu W., Gao G. F.: Changes in the length of the neur- 
aminidase stalk region impact H7N9 virulence in mice. J. Virol. 2016, 90, 2142-2149.

4. Campitelli L., Mogavero E., De Marco M. A., Delogu M., Puzelli S., Frezza F., Facchini M., Chiapponi C., Foni E., Cordioli P., Webby R., Barigazzi G., Webster R. G., Donatelli I.: Interspecies transmission of an H7N3 influenza virus from wild birds to intensively reared domestic poultry in Italy. Virology 2004, 323, 24-36.

5. Carnaccini S., Perez D. R.: H9 influenza viruses: an emerging challenge. Cold Spring Harb. Perspect. Med. 2020, 10, a038588.

6. Cheng J., Zhang C., Tao J., Li B., Shi Y., Liu H.: Effects of the $\mathrm{S} 42$ residue of the H1N1 swine influenza virus NS1 protein on interferon responses and virus replication. Virology J. 2018, 15, 57.

7. Cui L., Liu D., Shi W., Pan J., Qi X., Li X., Guo X., Zhou M., Li W., Li J., Haywood J., Xiao H., Yu X., Pu X., Wu Y., Yu H., Zhao K., Zhu Y., Wu B., Jin T., Shi Z., Tang F., Zhu F., Sun Q., Wu L., Yang R., Yan J., Lei F., Zhu B., Liu W., Ma J., Wang H., Gao G. F.: Dynamic reassortments and genetic heterogeneity of the human-infecting influenza A (H7N9) virus. Nat. Commun. 2014, 5, 3142 .

8. Fang S., Bai T., Yang L., Wang X., Peng B., Liu H., Geng Y., Zhang R., Ma H., Zhu W., Wang D., Cheng J., Shu Y.: Sustained live poultry market surveillance contributes to early warnings for human infection with avian influenza viruses. Emerg. Microbes Infect. 2016, 5, e79.

9. Li J., Ishaq M., Prudence M., Xi X., Hu T., Liu Q., Guo D.: Single mutation at the amino acid position 627 of PB2 that leads to increased virulence of an $\mathrm{H} 5 \mathrm{~N} 1$ avian influenza virus during adaptation in mice can be compensated by multiple mutations at other sites of PB2. Virus Res. 2009, 144, 123-129.

10. Li W., Wang G., Zhang H., Xin G., Zhang D., Zeng J., Chen X., Xu Y., Cui Y., $\mathrm{Li} K$.: Effects of NS1 variants of H5N1 influenza virus on interferon induction, TNF $\alpha$ response and p53 activity. Cell Mol. Immunol. 2010, 7, 235-242.

11. Lloren K. K. S., Taehyung L., Jung K. J., Min-Suk S.: Molecular markers for interspecies transmission of avian influenza viruses in mammalian hosts. Int. J. Mol. Sci. 2017, 18, 2706.

12.Lu S., Zhao Z., Zhang J., Wang W., He X., Yu M., Zhang C., Li X., Guo Z., Yang X., Liu L., Zhi M., Fu T., Lv X., Ma W., Liao M., Chai H., Liu L., Qian J., $M a J$. : Genetics, pathogenicity and transmissibility of novel reassortant H5N6 highly pathogenic avian influenza viruses first isolated from migratory birds in western China. Emerg. Microbes Infect. 2018, 7, 6 .

13. Ma M., Pau J., Geoff J., Williams B.: A report of birds banding and recovery in Xinjiang, China. Zoological Research 2002, 23, 105-106.

14. Matsuoka Y., Swayne D. E., Thomas C., Rameix-Welti M. A., Naffakh N., Warnes C., Altholtz M., Donis R., Subbarao K.: Neuraminidase stalk length and additional glycosylation of the hemagglutinin influence the virulence of influenza H5N1 viruses for mice. J. Virol. 2009, 83, 4704-4708.

15. Mosaad Z., Arafa A., Hussein H. A., Shalaby M. A.: In silico thermodynamic stability of mammalian adaptation and virulence determinants in polymerase complex proteins of H9N2 virus. J. Genet. Eng. Biotechnol. 2018, 16, 757-767.

16. Munier S., Larcher T., Cormier-Aline F., Soubieux D., Su B., Guigand L., Labrosse B., Cherel Y., Quéré P., Marc D., Naffakh N.: A genetically engineered waterfowl influenza virus with a deletion in the stalk of the neuraminidase has increased virulence for chickens. J. Virol. 2010, 84, 940-952.

17. Neumann G., Kawaoka Y.: Host range restriction and pathogenicity in the context of influenza pandemic. Emerg. Infect. Dis. 2006, 12, 881-886.

18. Pabbaraju K., Tellier R., Wong S., Li Y., Bastien N., Tang J. W., Drews S. J., Jang Y., Davis C. T., Fonseca K., Tipples G. A.: Full-genome analysis of avian influenza A(H5N1) virus from a human, North America, 2013. Emerge Infect. Dis. 2014, 20, 887-891.

19. Rolling T., Koerner I., Zimmermann P., Holz K., Haller O., Staeheli P., Kochs G.: Adaptive mutations resulting in enhanced polymerase activity contribute to high virulence of influenza A virus in mice. J. Virol. 2009, 83, 6673-6680.

20. Seo S. H., Hoffmann E., Webster R. G.: Editorial note: Lethal H5N1 influenza viruses escape host antiviral cytokine responses. Nat. Med. 2012, 18, 1592.

21. Shen Y. Y., Ke C. W., Li Q., Yuan R. Y., Xiang D., Jia W. X., Yu Y. D., Liu L., Huang C., Qi W. B., Sikkema R., Wu J., Koopmans M., Liao M.: Novel reassortant avian influenza A(H5N6) viruses in humans, Guangdong, China, 2015. Emerg Infect Dis. 2016, 22, 1507-1509.

22. Subbarao E. K., London W., Murphy B. R.: A single amino acid in the PB2 gene of influenza A virus is a determinant of host range. J. Virol. 1993, 67, 1761-1764.

23.Sun H., Pu J., Wei Y., Sun Y., Hu J., Liu L., Xu G., Gao W., Li C., Zhang X., Huang Y., Chang K. C., Liu X., Liu J.: Highly pathogenic avian influenza
H5N6 viruses exhibit enhanced affinity for human type sialic acid receptor and in-contact transmission in model ferrets. J. Virol. 2016, 24, 6235-6243.

24. Tong S., Li Y., Rivailler P., Conrardy C., Castillo D. A., Chen L. M., Recuenco S., Ellison J. A., Davis C. T., York I. A., Turmelle A. S., Moran D., Rogers S., Shi M., Tao Y., Weil M. R., Tang K., Rowe L. A., Sammons S., Xu X., Frace M., Lindblade K. A., Cox N. J., Anderson L. J., Rupprecht C. E., Donis $R$. $O$.: A distinct lineage of influenza A virus from bats. Proc. Natl. Acad. Sci. USA 2012, 109, 4269-4274.

25. Wang J., Wu M., Hong W., Fan X., Chen R., Zheng Z., et al.: Infectivity and transmissibility of avian H9N2 influenza viruses in pigs. J. Virol. 2016, 90, 3506-3514.

26. Wong F. Y., Phommachanh P., Kalpravidh W., Chanthavisouk C., Gilbert J., Bingham J., Davies K. R., Cooke J., Eagles D., Phiphakhavong S., Shan S., Stevens V., Williams D. T., Bounma P., Khambounheuang B., Morrissy C., Douangngeun B., Morzaria S.: Reassortant highly pathogenic influenza A(H5N6) virus in Laos. Emerg. Infect. Dis. 2015, 21, 511-516.

27. Ye Z. X., Liu H. X., Chen Y. N., Shu S., Wu Q. S., Wang S. J.: Analysis of water level variation of lakes and reservoirs in Xinjiang, China using ICESat laser altimetry data (2003-2009). Plos One 2017, 12, e0183800.

28. Zhang C., Zhao Z., Guo Z., Zhang J., Li J., Yang Y., Lu S., Wang Z., Zhi M., Fu Y., Yang X., Liu L., Zhang Y., Hua Y., Liu L., Chai H., Qian J.: Amino acid substitutions associated with avian H5N6 influenza A virus adaptation to mice. Front Microbe 2017, 8, 1763.

29. Zhou B., Donnelly M. E., Schole D. T., St George K., Kawaoka Y., Wentworth $D$. E.: Single-reaction genomic amplification accelerates sequencing and vaccine production for classical and swine origin human influenza A viruses. J. Virol. 2009, 83, 10309-10313.

Corresponding author: Prof. Zhenghai Ma, College of Life Science and Technology, Xinjiang University, Shengli road 666, Urumqi, Xinjiang, 830046, China; e-mail: mzhxju@126.com 